\title{
A DSMC Investigation of Gas Flows in Micro-Channels With Bends
}

\author{
Craig White*, Matthew K. Borg, Thomas J. Scanlon, Jason M. Reese \\ Department of Mechanical and Aerospace Engineering, University of Strathclyde, \\ Glasgow G1 1XJ, UK
}

\section{Abstract}

Pressure-driven, implicit boundary conditions are implemented in an open source direct simulation Monte Carlo (DSMC) solver, and benchmarked against simple micro-channel flow cases found in the literature. DSMC simulations are then carried out of gas flows for varying degrees of rarefaction along micro-channels with both one and two ninety-degree bends. The results are compared to those from the equivalent straight micro-channel geometry. Away from the immediate bend regions, the pressure and Mach number profiles do not differ greatly from those in straight channels, indicating that there are no significant losses introduced when a bend is added to a micro-channel geometry. It is found that the inclusion of a bend in a micro-channel can

\footnotetext{
${ }^{*}$ Corresponding author

Email addresses: craig.white@strath.ac.uk (Craig White), matthew.borg@strath.ac.uk (Matthew K. Borg), tom.scanlon@strath.ac.uk (Thomas J. Scanlon), jason.reese@strath.ac.uk (Jason M. Reese) 
increase the amount of mass that a channel can carry, and that adding a second bend produces a greater mass flux enhancement. This increase happens within a small range of Knudsen number $\left(0.02 \leq K n_{\text {in }} \leq 0.08\right)$. Velocity slip and shear stress profiles at the channel walls are presented for the Knudsen showing the largest mass flux enhancement.

Keywords: DSMC, micro-channel, bend, transition regime, rarefied gas dynamics

\section{Introduction}

Gaseous flows in micro-scale geometries are an increasingly important area of research for the design of micro-electro-mechanical system (MEMS) technologies such as micro-pumps, micro-turbines and micro-fuel cells. Gas flows at the micro-scale behave differently than predicted by macroscopic theory, mainly due to the combination of high gas rarefaction and the greatly increased importance of gas-surface interactions. The micro-metre length scales involved can make the continuum fluid assumption invalid altogether, and non-equilibrium effects, such as velocity slip, temperature jump and thermal transpiration, may have to be accounted for [1]. The degree of gas rarefaction is normally defined through the Knudsen number, 


$$
K n=\frac{\lambda}{L}
$$

where $\lambda$ is the mean free path of the gas molecules or atoms and $L$ is a characteristic length scale of the flow system.

The continuum fluid assumption is normally valid for $K n \leq 10^{-3}$, but can be extended as far as $K n \leq 10^{-1}$ with the introduction of velocity slip and temperature jump boundary conditions. Once $K n \geq 10^{-1}$, it is necessary to solve the Boltzmann equation to account for rarefaction effects properly. However, this proves difficult if $K n<10$ as inter-molecular collisions are still important and the Boltzmann collision integral term is very expensive to solve. A gas flow can be considered collisionless when $K n>10$ (the freemolecular regime) and so the Boltzmann equation can be solved for these kinds of flows.

MEMS devices involving gas flows tend to operate in the slip $\left(10^{-3} \leq\right.$ $\left.K n \leq 10^{-1}\right)$ and transition regimes $\left(10^{-1} \leq K n \leq 10\right)$, even at relatively high pressures (i.e. around atmospheric). It is the transition Knudsen number regime that presents the greatest difficulty to model numerically: the mean free path is significant compared to the characteristic length scale, and molecular collisions remain important. 
A variety of non-intuitive physics in high Knudsen number flows has been observed experimentally and can be predicted both analytically and numerically. For example, non-linear pressure gradients have been observed in micro-channel flows [2], along with relatively large velocity slip and temperature jump values. The movement of radiometer vanes when exposed to sunlight is caused by rarefied gas flow effects, i.e. uneven gas heating over a region bounded by around one mean free path length from the edges of the vanes, with gas "creeping" from a colder region towards a hotter region [3]. Non-equilibrium Knudsen layer effects are also important [4].

The direct simulation Monte Carlo (DSMC) method [5], based on the kinetic theory of dilute gases, has emerged as the dominant numerical technique for solving gas flows in the transition regime. There is plentiful DSMC data available for straight micro-channels [6-11]; contrastingly, there is a real absence of any data for micro-channels with bends. Micro-channels in practical applications tend to have a large number of bends, so there is a clear need for DSMC data on what effect bends can have on a micro-channel flow in the slip and transition Knudsen number regimes. A comprehensive review of gas flows in micro-channels can be found in Ref. [12].

In this paper we carry out DSMC simulations of micro-channels with 
bends. In $\S 2$ we describe the DSMC algorithm and pressure boundary conditions to be applied to the micro-channels. In $\S 3$ we benchmark the boundary conditions against simple cases in the literature. In $\S 4$ and $\S 4.2$ we apply the newly implemented boundary conditions to micro-channels with bends.

\section{Numerical Technique}

\subsection{Direct simulation Monte Carlo}

Originally developed to solve rarefied gas flows in low density, hypersonic problems, the DSMC method has more recently been applied to MEMS applications. The basic premise of the method is that a single DSMC simulator particle can represent any number of real atoms or molecules - vastly reducing the computational expense required to run a simulation - and that particle movements and collisions can be decoupled. The time step size must be smaller than the mean collision time (the average time between successive collisions of a particle) to allow for successful decoupling of the movement and collision steps. The particles are moved ballistically in the computational domain according to their individual velocity vectors and the time step size. Interactions with any boundaries (e.g. introduction of new particles, collisions with surfaces, passing from one processor to another) are dealt with 
during the movement step. Inter-particle collisions, and the associated exchange of energy between different modes, are considered stochastically after all particles have been moved. Finally, the microscopic particle properties are sampled and used to recover the relevant macroscopic data. A detailed description of the DSMC algorithm can be found in [5].

Micro-channel gas flows are often characterised as being low speed and operating around atmospheric pressure. This can present problems when trying to recover macroscopic fields such as temperature and velocity from DSMC simulations. The thermal velocities of the particles are likely to be at least an order of magnitude larger than the macroscopic velocity and this makes it statistically difficult to recover the macroscopic velocity. A large sample size is required to reduce the statistical error in the measurement of macroscopic fields to an acceptable level. Simulations involving very low velocities $(\mathrm{Ma}<0.01)$ are often impractical as the required sample sizes would simply be too large [13]. This problem of statistical scatter restricts particle methods such as DSMC to the study of shorter micro-channels operating at much higher velocities than experimental micro-channels.

The DSMC code used in the present work has been implemented in OpenFOAM, an open-source $\mathrm{C}++$ tool box for computational fluid dynamics 
which is free to download from [14]. This solver, which we called dsmcFoam, has been rigorously validated for a variety of benchmark cases $[15,16]$, mostly concerning hypersonic flows. In the current work, a set of sub-sonic, implicit pressure boundary conditions has been implemented (as described in $\S 2.2$ ) within dsmcFoam.

\subsection{Pressure Boundary Conditions}

Gas flows in MEMS are characteristically low speed and driven by a pressure gradient. Unlike more traditional hypersonic DSMC simulations, it is likely that particles will have thermal velocities orders of magnitude larger than the stream velocity. This stochastic motion makes it likely that particles do not flow uninterrupted in the streaming direction, but instead may, for example, exit at the inlet or enter at the outlet. The velocity profiles at the inlet and outlet are not known due to current experimental limitations at the micro-scale. Pressure and temperature are usually the only known thermo-mechanical properties at the boundaries.

Piekos \& Breuer [17] proposed pressure boundary conditions for low speed flows in micro-channels, intended to be applied far from the high and low pressure reservoirs, and they achieved excellent agreement between their DSMC results and an analytical solution in the slip flow regime for a straight micro- 
channel of aspect ratio 30. Nance et al. [6] developed a set of boundary conditions suitable for DSMC simulation of low speed flows in micro-channel geometries by adapting computational fluid dynamics (CFD) theory of characteristics boundary conditions at the exit boundary, and using particle flux conservation at the inlet. They found that elongated cell sizes in the streamwise direction provided solutions consistent with much smaller (i.e. fraction of the mean free path) cell sizes. The macroscopic gradients in low speed flows tend to be small in the stream-wise direction, allowing for a large decrease in the computational expense of a micro-channel simulation. Again, only straight micro-channel geometries were considered in that work.

Wu et al. [18] developed low speed boundary conditions using particle flux conservation at both inlet and outlet boundaries, and applied them to geometries that were not simply straight micro-channels. For example, they simulated argon gas flow in a pressure-driven T-junction geometry with a single inflow boundary and two exit boundaries [19].

Liou \& Fang $[7,8,20]$ improved the Nance et al. boundary conditions by removing the particle flux dependence at the inlet and replacing it with a first order extrapolation and time average of the streamwise velocity from the boundary cells. The theory of characteristics outlet boundary remained 
unchanged from the Nance et al. implementation. This improved convergence speed at the inlet boundaries by reducing the statistical scatter. A variety of benchmark cases for straight micro-channels with aspect ratio 5 were presented. Wang \& Li [21] further improved the convergence behaviour of these boundary conditions by applying the theory of characteristics at the inlet in addition to the outlet. They also presented results for flow in a micro-channel with a single ninety-degree bend, but there was no detailed study of micro-channels with bends.

More recently, Ye et al. $[10,11,22]$ proposed boundaries similar to those of Nance et al. [6] and Wang \& Li [21], but they used different correction factors at the inlets and outlets in order to improve the heat transfer results in micro-channels whose surface temperature is substantially different from the gas inflow temperature.

The general starting point for the treatment of these boundary conditions is to impose a particle flux at an inlet or outlet boundary. The rate of particle insertion, $\dot{N}$, can be computed from the equilibrium Maxwell-Boltzmann distribution [5]:

$$
\frac{\dot{N}}{A}=\frac{n V_{m p}\left\{\exp \left(-q^{2}\right)+\sqrt{\pi} q[1+\operatorname{erf}(q)]\right\}}{2 \sqrt{\pi}}
$$


where

$$
\begin{gathered}
q=\frac{V}{V_{m p}} \cos \theta, \\
V_{m p}=\sqrt{\frac{2 k T}{m}},
\end{gathered}
$$

and $\theta$ is the angle to the surface normal, $A$ is the boundary area, $V$ is the local stream velocity, $V_{m p}$ is the local most probable thermal velocity, and $n$ is the number density at the boundary, $T$ is the macroscopic temperature at the boundary, $m$ is the molecular mass and $k$ is the Boltzmann constant. It is not always certain that the velocity distribution function will be Maxwellian at the inlet or outlet of a micro-channel, but it is also not easy to define what form the actual distribution function will have. For this reason, these boundary conditions assume a Maxwellian form. The streaming velocity profile at the inlet is generally not known, so we must rely on boundary values of temperature, density and pressure to derive the corresponding local velocity required for Equation (2). The boundary conditions used in the present work are those proposed by Wang \& Li [21]. 


\section{Benchmarking}

\subsection{Micro-Poiseuille Flow}

This benchmark case is the micro-channel Poiseuille flow of nitrogen gas. The geometry is a channel of height $0.4 \mu \mathrm{m}$, and $2 \mu \mathrm{m}$ length, meshed with $100 \times 60$ computational cells. The surface and inlet gas temperatures are set to $300 \mathrm{~K}$. The outlet pressure is atmospheric, and the inlet to outlet pressure ratio is 2.5. The Knudsen number at the inlet is 0.055 and 0.123 at the exit. The simulation contained around 180,000 DSMC simulator particles and was solved in parallel on 2 processors. A total of 425,000 samples required a run time of 19 hours.

Figure 1 shows our results from dsmcFoam compared with previous numerical work of the same case from both Liou \& Fang [7] and Roohi et al. [9]. A first order analytical slip solution is also provided for reference. Good agreement between the DSMC data and seemingly good agreement with the continuum solution, can be seen in Figure 1(a). The relative deviation from a conventional linear pressure drop along the channel centre-line is shown in Figure 1(b). Good agreement is found with the results published in $[9]$. 


\subsection{Micro-Manifold Flow}

The flow of argon gas through a T-junction micro manifold, which was investigated first by Wu \& Tseng [19], is studied here. A diagram of the geometry is shown in Figure 2, where the height $h$ is $1 \mu \mathrm{m}, H$ is $3 \mu \mathrm{m}$, and the length $L$ is $7 \mu \mathrm{m}$. In this case the geometry has two outlet channels and one inlet channel that require separate treatment of pressure boundary conditions. Three different pressure configurations are tested, as detailed in Table 1 , where $p_{i}$ is the inlet pressure, and $p_{e 1}$ and $p_{e 2}$ are the outlet pressures. The working gas is argon; all surface temperatures and gas inlet temperatures are constant at $300 \mathrm{~K}$. The inlet Knudsen number, based on the VHS mean free path and the micro-manifold height, is 0.2 . The time step employed was $8.33 \times 10^{-11}$ s for all cases. Our dsmcFoam results use the boundary conditions described above, but the $\mathrm{Wu} \&$ Tseng results used boundary conditions which employ particle flux conservation at both inlets and outlets.

Table 2 lists the results for the normalised mass flow rates at each inlet and outlet boundary. All of the mass flux results from dsmcFoam exhibit less than $0.01 \%$ statistical uncertainty based on the standard error from the standard deviation of the instantaneous samples. The Wu \& Tseng results are displayed in standard text and the $d s m c F o a m$ results are presented 
below them in bold font. The summation column has been calculated as $\left(\dot{m}_{e 1}+\dot{m}_{e 2}\right) / \dot{m}_{i}$ and the error as $\left(\dot{m}_{e 1}+\dot{m}_{e 2}-\dot{m}_{i}\right) / \dot{m}_{i}$

Good agreement has been found with the results of Wu \& Tseng. The summation values from $d s m c F o a m$ are closer to unity than the Wu \& Tseng results and so the percentage errors in the conservation of mass are as much as two orders of magnitude smaller for our dsmcFoam results.

Figures 3 and 4 compare contours of pressure and Mach number for Case I from dsmcFoam with those presented by Wu \& Tseng [19]. Excellent agreement has been found in both cases. The dsmcFoam simulation contained around 285,000 DSMC particles at steady state, and the results were averaged for 590,000 time steps in parallel on 6 cores for a total run time of around 64 hours, and exhibit much less scatter than the Wu \& Tseng results.

\section{Micro-Channels with Bends}

\subsection{Previous Numerical Work}

There has been very little numerical work concerning micro-channels with bends in the slip and transition regimes, despite this being a very important area in the design of MEMS. The only work which has explicitly dealt with this kind of problem using DSMC is that of Wang \& Li [21]. One of their 
ninety-degree bend micro-channel cases is repeated here, and some improvements proposed on how to mesh these types of geometries in order to capture some surprising and significant flow features.

The geometry is shown in Figure 5, where $L$ is $5 \mu \mathrm{m}$ and $H$ is $1 \mu \mathrm{m}$. The working gas is nitrogen and the wall and inlet gas temperatures are all equal at $300 \mathrm{~K}$. In Ref. [21] it is stated that $100 \times 100$ rectangular computational cells were used to mesh the geometry. A different mesh is used in the current work: $200 \times 60$ rectangular cells in each arm of the channel, with $60 \times$ 60 square cells in the corner region. This has been done to achieve a higher resolution of macroscopic properties in the corner region. The outlet pressure is $100 \mathrm{kPa}$ and the inlet to outlet pressure ratio is 3. This gives Knudsen numbers ranging from 0.018 to 0.055 along the length of the micro-channel. The dsmcFoam simulation contained 1.6 million DSMC particles at steady state, and over 1 million samples were employed to be consistent with the work in Ref. [21].

A comparison of mass density contours with velocity streamlines is shown in Figure 6. The values of the contours are not given in [21], so it has not been possible to use the exact same values for the $d s m c F o a m$ results. In general, good qualitative agreement can be found between the two cases. It 
is stated by Wang \& $\mathrm{Li}[21]$ that no flow separation was found at the corner, however dsmcFoam has captured a region of separated flow - a close up view of this separation can be seen in Figure 7. Some experimental work in micro-channels with bends found evidence that there may be separation zones in the corner regions $[23,24]$. Considerable evidence of flow separation has been found in related geometries operating at similar Reynolds and Knudsen numbers, such as constriction micro-channels [25] and backwards facing steps [26]. The mechanism for this flow separation is not well understood. Interestingly, the flow from our DSMC results does not exhibit the features of Stokes flow (i.e. no separation even at sharp corners) that would be expected at the low Reynolds numbers under consideration.

It is probable that the cell size used in [21] is simply too large to capture the relatively small macroscopic gradients of the separation zone. Although it is possible to use elongated cell sizes in the streamwise direction of microchannel flows [6], care must be taken when a bend is introduced to ensure that cell sizes are small enough to capture the larger gradients present in any separation zones near the corners.

The micro-channel considered here remains entirely in the slip flow Knudsen number regime. There is no DSMC data for this kind of geometry in the 
transition regime, but there is some lattice Boltzmann data [27] that extends into the transition regime, achieving a maximum Knudsen number of 0.286. The geometry considered there is a micro-channel with a single ninety-degree bend and a larger aspect ratio than that of [21]. Flow separation at the corners was seen in the lattice Boltzmann results, and the size of the separation regions decreased with increasing Knudsen number.

\subsection{DSMC Investigation of Micro-Channels with Bends}

We focus on providing DSMC data in the late slip and early transition Knudsen number regimes. Micro-channel geometries with both one and two ninety-degree bends are considered and compared to results from a straight channel, as shown in Figure 8: the total flow length $L$ is the same for all three geometries to ensure a fair comparison. Mass fluxes are calculated, and pressure and Mach number profiles along the micro-channel centre-lines are plotted for a range of Knudsen and Reynolds numbers. Each case contained approximately one million DSMC simulator particles and required one week of sampling in parallel on 8 cores to achieve a sufficient number of statistical samples, i.e. in excess of one million.

Table 3 details the inlet and outlet pressures for each case we considered, along with values for the time step, and the Knudsen, Reynolds and Mach 
numbers at the inlet ("in") and outlet ("out"). The working gas is argon, set at $300 \mathrm{~K}$ at the inlet for all cases. All walls are modelled as fully diffuse boundaries (i.e. the tangential momentum accommodation coefficient is 1.0) at $300 \mathrm{~K}$. The cases cover a range of situations: Cases 1 and 2 are completely in the slip regime, in Cases 3 and 4 the inlet flow is the slip regime and the outlet flow is in the transition regime, and Cases 5 and 6 are entirely in the transition regime. A slightly smaller time step has been used for the case at the highest pressure to ensure that the time step remained much smaller than the mean collision time. The values of $K n_{\text {out }}, R e_{\text {out }}$ and $M a_{\text {out }}$ were calculated during post-processing at the exit centre-line: $K n_{\text {out }}$ was calculated using the local temperature and density with the VHS mean free path, and $R e_{\text {out }}$ and $M a_{\text {out }}$ calculated using the following formulations:

$$
R e_{\text {out }}=\frac{U_{\text {out }} \rho_{\text {out }} H}{\mu_{\text {out }}}
$$

and

$$
M a_{\text {out }}=\frac{U_{\text {out }}}{\sqrt{\gamma R T_{\text {out }}}}
$$

where $\gamma$ is the ratio of specific heats and $\mu$ is the local gas viscosity. Figure 8 is a diagram of the three geometries considered here, where $L$ is $15 \mu \mathrm{m}$ and $H$ is $1 \mu \mathrm{m}$. It should be noted that all of the current cases are two-dimensional, effectively representing a cross-section of a wide micro-channel. 
The normalised centre-line pressure distributions calculated in each of the six cases are shown in Figure 9. In Figure 9(a), the profiles in the straight micro-channel geometries are presented. As expected, non-linear pressure profiles have been found, and the degree of non-linearity decreases with increasing Knudsen number as rarefaction effects $(K n)$ begin to dominate the compressibility effects $(M a)$.

Figure 9(b) shows the normalised pressure profiles for the channel with a single ninety-degree bend. The pressure profiles differ from the straight channel by having noticeable jumps in local pressure at the bend location. These jumps are compressibility effects, which are introduced by a reduction of the gas velocity in the flow direction, due to the abrupt changes in channel geometry. For cases of lower Mach number, however, the compressibility effects are less, and proportionally so are the pressure jumps at the bend locations. Figure 9(c) shows very similar behaviour for a micro-channel with two ninety-degree bends, instead with the pressure having two peaks. This analysis is further verified by the Mach number plots shown in Figure 10; local Mach number decreases significantly at bend locations.

To illustrate how closely the pressure and Mach number profiles match outside of the bend regions, Figure 11 compares the profiles of pressure and 
Mach number for the different geometries when the inlet Knudsen number is 0.011 . Away from the the bends, the pressure profiles all match well and the degree of non-linearity is maintained. This is in contrast to constriction micro-channel flows where the pressure profile is entirely different from that of the equivalent straight micro-channel [25]. The Mach number profiles in Figure 11(b) show a similar pattern, with all three cases reaching very nearly the same Mach number at the exit and the profiles being of very similar shape outside of the bend regions. The similarity of the pressure and Mach number profiles found between the bent and straight channel geometries suggests that there are no significant pressure losses introduced by the inclusion of a bend, and consequently similar mass fluxes should be expected. However, the lattice Boltzmann results in Ref. [27] indicate that the presence of a bend may result in a modest increase in the mass-carrying capacity of a micro-channel.

To investigate this phenomenon using DSMC, we plot the mass fluxes in Figure 12 for the single bend and double bend micro-channel geometries. Each of the data points has been normalised with the mass flux from the corresponding straight micro-channel. The measured error in all of the mass flux values is less than $0.5 \%$, based on the standard error from the standard deviation of the instantaneous samples. The error bars associated with the 
data points are much smaller than the plotted points and have therefore been omitted. We define the percentage flow enhancement, $\epsilon$, as

$$
\epsilon=100\left[\frac{\left(\dot{m}_{\text {bend }}-\dot{m}_{\text {straight }}\right)}{\dot{m}_{\text {straight }}}\right]
$$

where $\dot{m}_{\text {bend }}$ is the mass flux from the bent channel simulation and $\dot{m}_{\text {straight }}$ is the mass flux from its corresponding straight channel simulation.

In agreement with the lattice Boltzmann [27] and continuum [28] results, Figure $12(\mathrm{a})$ shows a relative increase of up to $1 \%$ in the amount of mass that a channel with a single bend can carry when $0.02 \leq K n_{\text {in }} \leq 0.08$. For the micro-channels with two ninety-degree bends, Figure 12(b) shows a maximum $1.7 \%$ flow enhancement, and roughly in the same range of Knudsen number. For the other Knudsen numbers considered in this work there is a negative flow enhancement for both types of bended channels when compared to the corresponding straight channel cases. For example, at $K n_{\text {in }}=0.3$, a $3 \%$ decrease in flux is found for the single bend, and a $6 \%$ decrease for the double bend channel.

Table 4 shows the DSMC results for the average shear stress at both channel walls for a single and double bend, normalised with the average shear stress at the walls of the equivalent straight channels. The shear stress was calculated from the pre- and post-interaction components of particle 
momentum tangential to the surface, as described in [20]. It is found that the inclusion of a bend in a micro-channel always reduces the average shear stress at the walls, and that adding a second bend results in a larger decrease. In systems where a positive mass flux enhancement is found (single bend, $K n_{\text {in }}=0.027,0.055$ and 0.09 ; double bend, $K n_{\text {in }}=0.027$ and 0.055$)$ the shear stress ratio is closer to unity than those in which a decrease is found.

Figure 13 shows the shear stress distribution at the upper and lower walls of a channel with a single bend at an inlet Knudsen number of 0.027 , the case with the largest mass flux enhancement. The shear stress distribution in the equivalent straight channel is plotted for reference. It is clear that most of the roughly $12 \%$ reduction in average shear stress in the channel with a bend comes at the outside of the bend on the lower wall. There is a small decrease in shear stress after the bend at the upper wall, but this is offset by a small increase immediately prior to the bend. The corresponding slip velocities are shown in Figure 14 and follow very similar patterns to the shear stress profiles. The decrease in shear stress in regions of lower slip velocity is expected from Maxwell's velocity slip relation, since $\left|\vec{u}_{\text {slip }}\right| \propto|\vec{\tau}|[1,29]$.

Figure 15 shows the differences between the centre-line pressure profiles along micro-channels with a single bend and a double bend, with respect 
to the equivalent straight micro-channels. Here, different behaviour can be observed at the Knudsen numbers where the positive and negative flow enhancements are found: for a micro-channel with a single bend, at the two Knudsen numbers where a small flow increase is observed $\left(K n_{\text {in }}=0.027\right.$ and 0.055$)$, the pressure difference from a straight micro-channel is negative before the bend and positive after it. At an inlet Knudsen number of 0.3, the opposite behaviour is found, i.e. positive difference before the bend and negative after it. For the remaining two Knudsen numbers where the mass flux is nearly the same as in the straight channel, there is very little difference in the pressure profiles outside of the bend region. Similar patterns are found for a micro-channel with two bends, with some different behaviour being evident between the two bends.

\section{Conclusions and Future Work}

The implementation of low-speed, pressure-driven, implicit boundary conditions for DSMC has been undertaken within the framework of the free and open-source DSMC solver dsmcFoam, and benchmarked for a variety of different test cases found in the literature. For a micro-channel with a bend, we have shown that the choice of mesh size in the corner region is important 
to capture the size and shape of any recirculation zones. However, DSMC without any variance reduction is perhaps not the best tool for attempting to capture these kinds of flow features, as the flow velocities can be extremely low in these regions, requiring prohibitively large sample sizes.

A clear need for DSMC data in micro-channels with bends has been identified, and some new data for this type of problem has been presented and discussed. The pressure and Mach number profiles along the centre-line of a micro-channel with a single or double bend are very similar (outside the immediate location of a bend) to that of an equivalent straight micro-channel for the range of Knudsen numbers investigated here. This suggests that there are no significant losses induced when a sharp ninety-degree bend is introduced to a micro-channel system. Indeed, a slight increase in mass flux, compared to that of an equivalent straight micro-channel can be achieved when a bend is introduced. Although this has been observed using continuum and lattice Boltzmann solvers previously, this is the first time the phenomenon has been observed using the more reliable DSMC method. This mass flux enhancement is limited to a small range of Knudsen number and outwith this range a decrease in mass flux is found, with the magnitude of the decrease increasing as the flow moves into the transition regime. Adding a second bend to the 
system enables a larger mass flux enhancement to be achieved, but also leads to larger mass flux decreases outside of the small enhancement regime. The small range of Knudsen number where an increase in mass flux is found could be helpful in the design of MEMS devices, such as micro-fuel cells, but may not be maintained for longer channels with larger pressure drops, since the mass flux enhancement is restricted to a small Knudsen number range.

Velocity slip and shear stress profiles at the channel walls show good correlation with one another, as would be expected from Maxwell's velocity slip relation. Normalised shear stress values closer to unity are found at Knudsen numbers with positive mass flux enhancements. The centre-line pressure deviation plots also show markedly different behaviour at the Knudsen numbers where positive enhancements are found compared to those with negative enhancements.

Future work in this area using DSMC could include extending the current analysis to channels with more bends to check if the enhancement trend is maintained. Adding curvature to the bends would enable investigation of what the effect of competing concave and convex curvature is on the flow. The Knudsen numbers should be extended towards the free-molecular regime to investigate how the mass flux enhancement varies. The length of the micro- 
channels could be extended in order to obtain a larger aspect ratio, closer to that likely to be found in any practical micro-channel. Extending the length would also reduce the signal-to-noise ratio, so there is a practical limit to DSMC being a viable tool to provide this kind of information. However, variance reduction techniques [30] offer the promise of extending particle techniques to very low speed flows.

\section{Acknowledgements}

Craig White was funded by a scholarship from the James Weir Foundation. Our calculations were performed on the 1100 core HPC Facility of the Faculty of Engineering at the University of Strathclyde.

\section{References}

[1] M. Gad-el-Hak. The fluid mechanics of microdevices - the Freeman Scholar lecture. Journal of Fluids Engineering, 121(1):5-33, 1999.

[2] E.B. Arkilic, K.S. Breuer, and M.A. Schmidt. Mass flow and tangential momentum accommodation in silicon micromachined channels. Journal of Fluid Mechanics, 437:29-43, 2001. 
[3] S.F. Gimelshein, N.E. Gimelshein, A.D. Ketsdever, and N.P. Selden. Analysis and applications of radiometeric forces in rarefied gas flows. AIP Conference Proceedings, 1333(1):693-700, 2011.

[4] D.A. Lockerby, J.M. Reese, and M.A. Gallis. The usefulness of higherorder constitutive relations for describing the Knudsen layer. Physics of Fluids, 17(10):100609, 2005.

[5] G.A. Bird. Molecular Gas Dynamics and the Direct Simulation of Gas Flows. Oxford Science Publications, Oxford University Press Inc, New York, 1994.

[6] R.P. Nance, D.B. Hash, and H.A. Hassan. Role of boundary conditions in Monte Carlo simulation of MEMS devices. Journal of Thermophysics and Heat Transfer, 12(3):119-128, 1997.

[7] W.W. Liou and Y. Fang. Heat transfer in microchannel devices using DSMC. Journal of Microelectromechanical Systems, 10(2):274-279, 2001.

[8] Y. Fang and W.W. Liou. Computations of the flow and heat transfer in microdevices using DSMC with implicit boundary conditions. Journal of Heat Transfer, 124(2):338-345, 2002. 
[9] E. Roohi, M. Darbandi, and V. Mirjalili. Direct simulation Monte Carlo solution of subsonic flow through micro/nanoscale channels. Journal of Heat Transfer, 131(9):092402, 2009.

[10] J.J. Ye, J. Yang, J. Y. Zheng, W.Z. Li, S.Z. He, and Y.B. Ma. New treatment of pressure boundary conditions for DSMC method in micro-channel flow simulations. ASME Conference Proceedings, 2007(42886):121-126, 2007.

[11] J.J. Ye, J. Yang, J.Y. Zheng, P. Xu, C. Lam, L. Wong, and Y. Ma. Effects of wall temperature on the heat and mass transfer in microchannels using the DSMC method. In Nano/Micro Engineered and Molecular Systems, 2009. NEMS 2009. 4th IEEE International Conference on, pages 666-671, 2009.

[12] A. Agrawal. A comprehensive review on gas flow in microchannels. International Journal of Micro-Nano Scale Transport, 2(1):1-40, 2011.

[13] N.G. Hadjiconstantinou, A.L. Garcia, M.Z. Bazant, and G. He. Statistical error in particle simulations of hydrodynamic phenomena. Journal of Computational Physics, 187(1):274-297, 2003.

[14] OpenFOAM Foundation. http://www.openfoam.org/, 2012. 
[15] T.J. Scanlon, E. Roohi, C. White, M. Darbandi, and J.M. Reese. An open source, parallel DSMC code for rarefied gas flows in arbitrary geometries. Computers \& Fluids, 39(10):2078-2089, 2010.

[16] E. Arlemark, G. Markelov, and S. Nedea. Rebuilding of Rothe's nozzle measurements with OpenFOAM software. Journal of Physics: Conference Series, 362:012040, 2012.

[17] E.S. Piekos and K.S. Breuer. Numerical modeling of micromechanical devices using the direct simulation Monte Carlo method. Journal of Fluids Engineering, 118(3):464-469, 1996.

[18] J.-S. Wu, F. Lee, and S.-C. Wong. Pressure boundary treatment in micromechanical devices using the direct simulation Monte Carlo method. JSME International Journal Series B Fluids and Thermal Engineering, 44(3):439-450, 2001.

[19] J.-S. Wu and K.-C. Tseng. Analysis of micro-scale gas flows with pressure boundaries using direct simulation Monte Carlo method. Computers \& Fluids, 30(6):711-735, 2001.

[20] W.W. Liou and Y. Fang. Microfluid Mechanics. McGraw-Hill, New York, 2006. 
[21] M. Wang and Z. Li. Simulations for gas flows in microgeometries using the direct simulation Monte Carlo method. International Journal of Heat and Fluid Flow, 25(6):975-985, 2004.

[22] J. Yang, J.J. Ye, J.Y. Zheng, I. Wong, C.K. Lam, P. Xu, R.X. Chen, and Z.H. Zhu. Using direct simulation Monte Carlo with improved boundary conditions for heat and mass transfer in microchannels. Journal of Heat Transfer, 132(4):041008, 2010.

[23] S.Y.K. Lee, M. Wong, and Y. Zohar. Gas flow in microchannels with bends. Journal of Micromechanics and Microengineering, 11(6):635-644, 2001.

[24] V. Varade, A. Pradeep, S. Prabhu, and A. Agrawal. Experimental study of rarefied gas flow through a 90 degree bend tube. In Proceedings of the 2nd European Conference on Microfluidics, 2010.

[25] A.A. Alexeenko, S.F. Gimelshein, and D.A. Levin. Reconsideration of low reynolds number flow-through constriction microchannels using the DSMC method. Journal of Microelectromechanical Systems, 14(4):847$856,2005$.

[26] A. Beskok, G.E. Karniadakis, and W. Trimmer. Rarefaction and com- 
pressibility effects in gas microflows. Journal of Fluids Engineering, 118(3):448-456, 1996.

[27] A. Agrawal, L. Djenidi, and A. Agrawal. Simulation of gas flow in microchannels with a single ninety-degree bend. Computers 86 Fluids, 38(8):1629-1637, 2009.

[28] O. Rovenskaya and G. Croce. Numerical analysis of rarefaction and compressibility effects in bent microchannels. ASME Conference Proceedings, 2010(54501):451-458, 2010.

[29] D.A. Lockerby, J.M. Reese, D.R. Emerson, and R.W. Barber. Velocity boundary condition at solid walls in rarefied gas calculations. Physical Review E, 70:017303, 2004.

[30] G.A. Radtke, N.G. Hadjiconstantinou, and W. Wagner. Low-noise Monte Carlo simulation of the variable hard sphere gas. Physics of Fluids, 23(3):030606, 2011. 


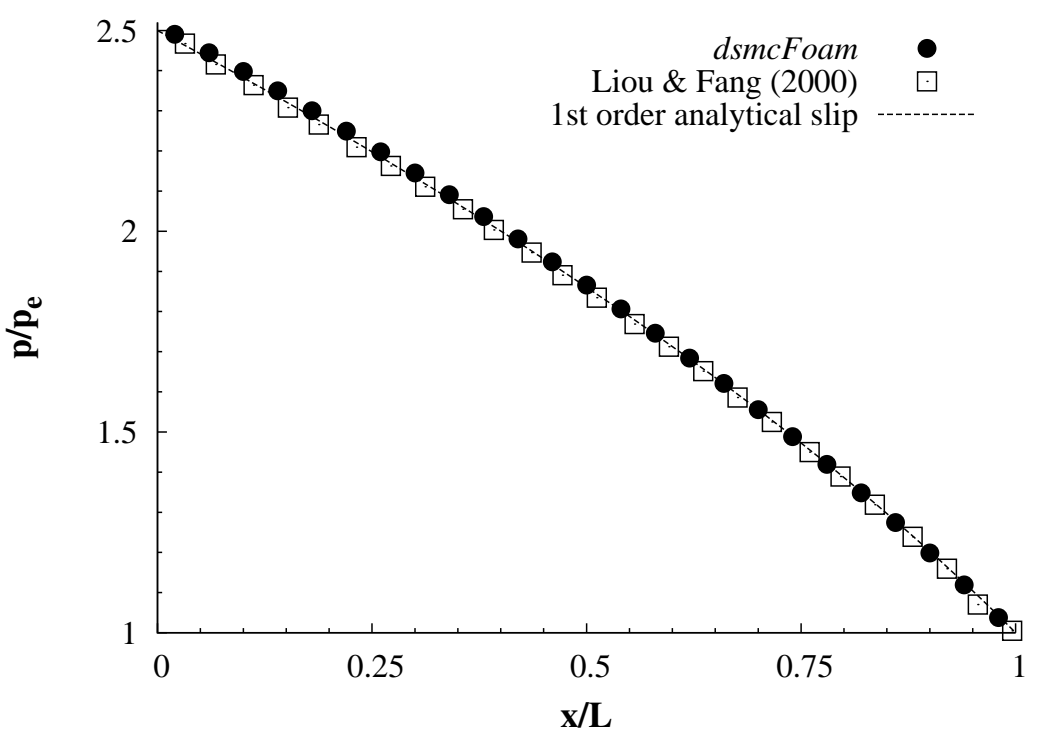

(a)

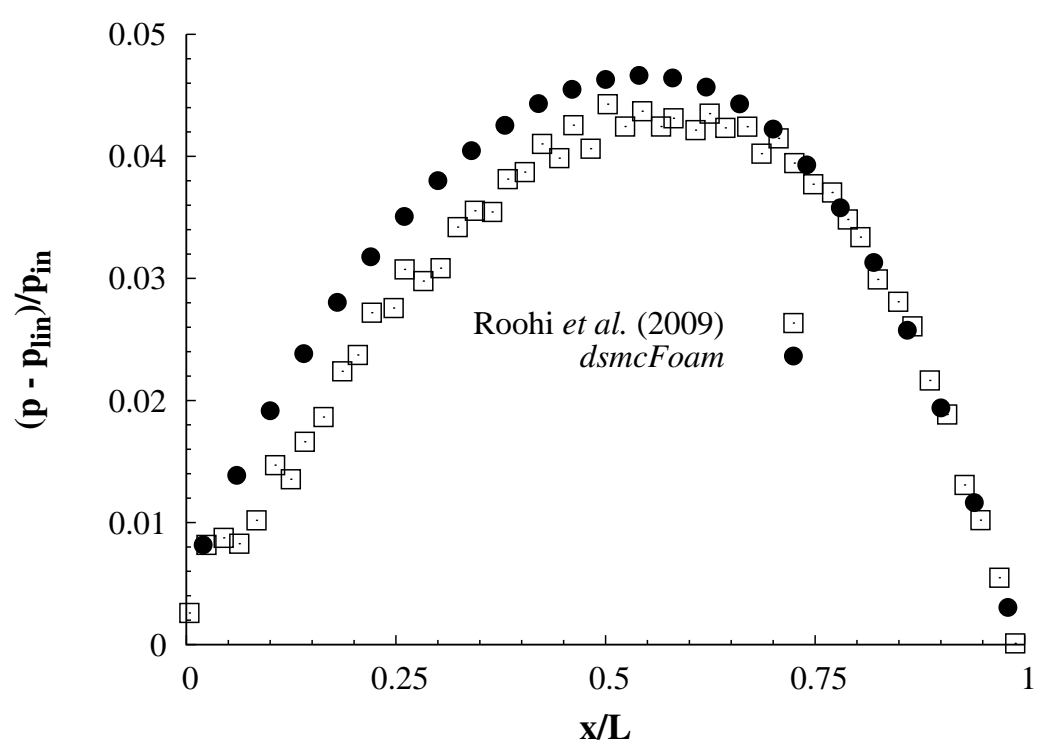

(b)

Figure 1: Comparisons with previous numerical work from $[7,9]$ for the micro-Poiseuille flow geometry for (a) streamwise centre-line pressure distribution, and (b) deviation of this distribution from a linear profile. 


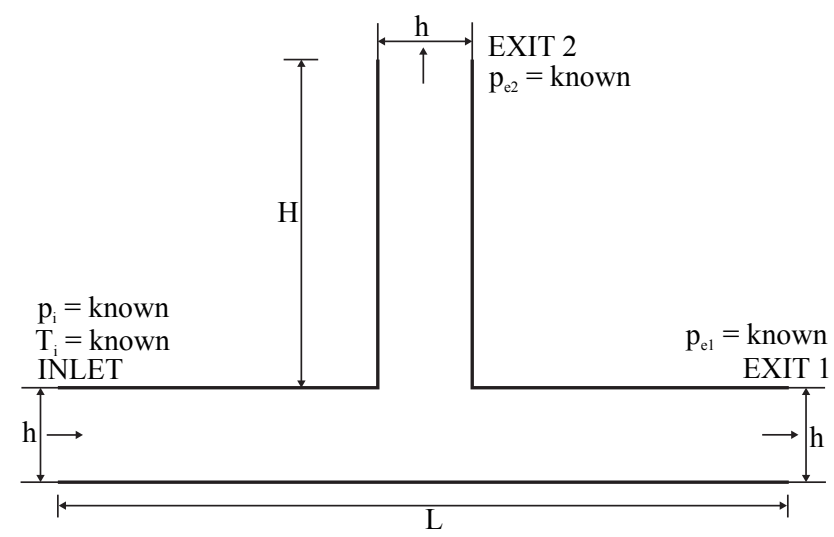

Figure 2: Schematic of the micro-manifold test case geometry [19]. 

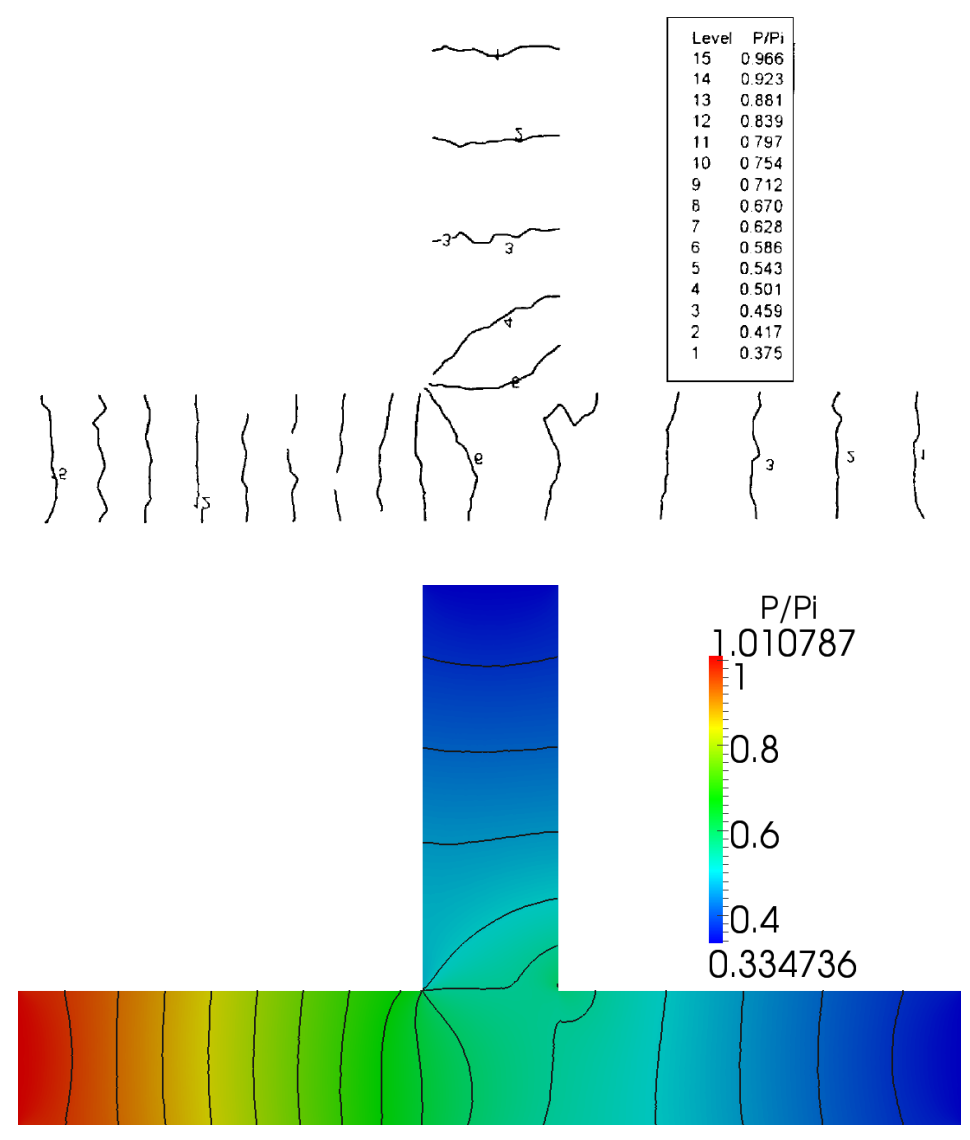

Figure 3: Micro-manifold flow: pressure contours for Case I. Top: Wu \& Tseng [19]; bottom: dsmcFoam. 

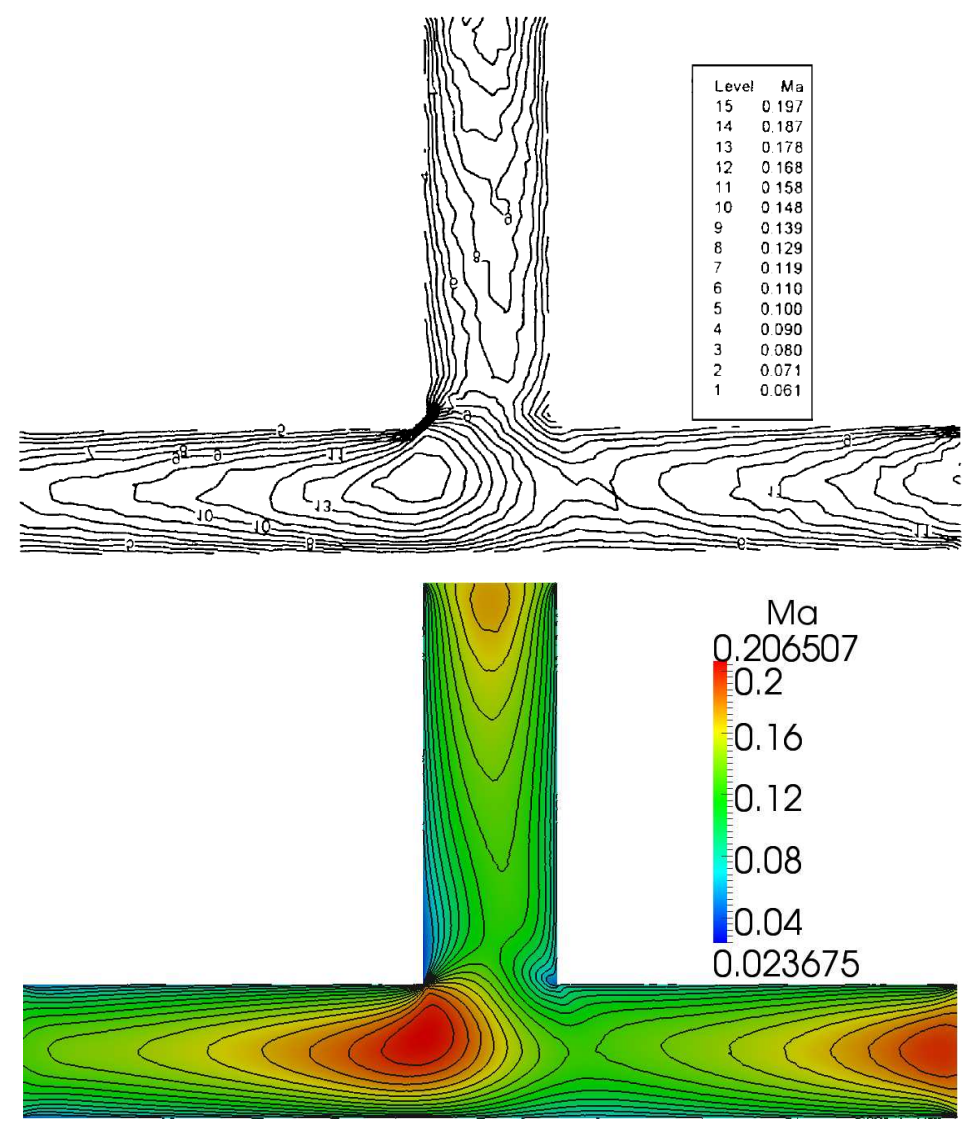

Figure 4: Micro-manifold flow: Mach number contours for Case I. Top: Wu \& Tseng [19]; bottom: dsmcFoam. 


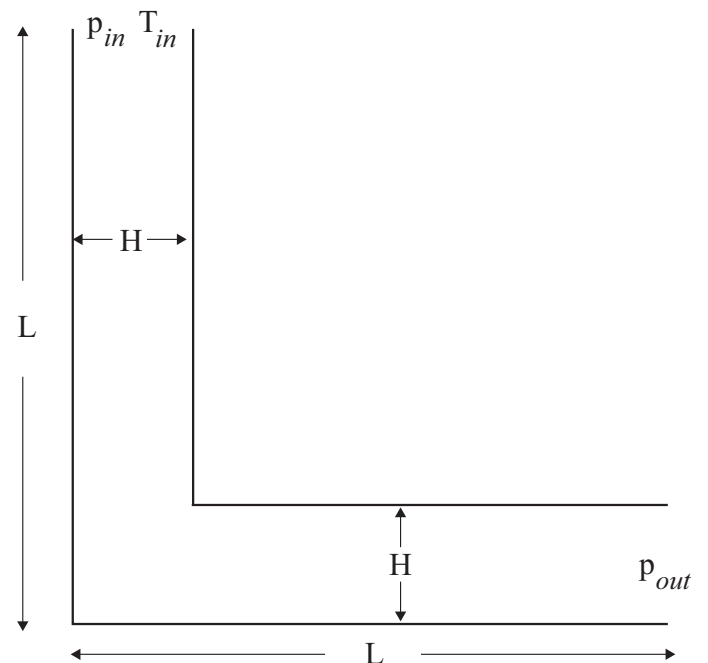

Figure 5: Schematic of the single ninety-degree bend micro-channel. 

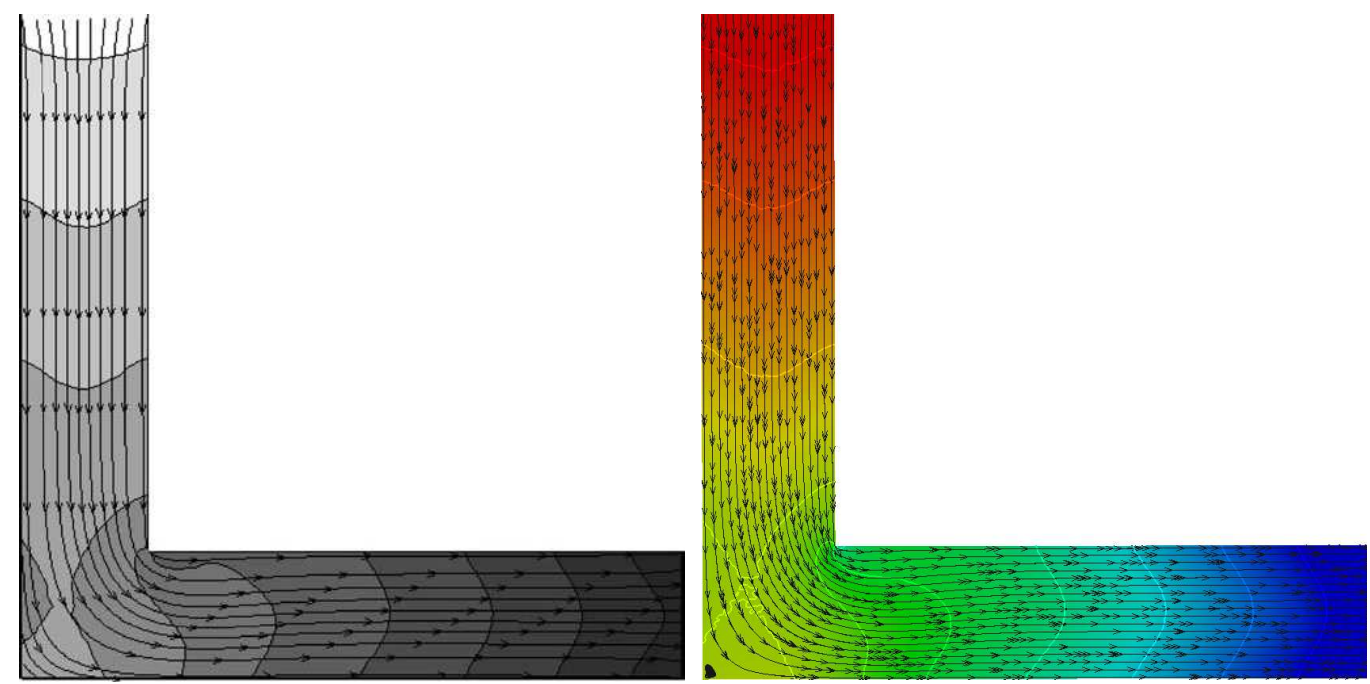

Figure 6: Comparison of mass density contours and velocity streamlines. Left: Wang and Li [21]; right: dsmcFoam results. Note the presence of a separated flow region in the corner region of the $d s m c F o a m$ results, as shown in enlarged view in Figure 7. 


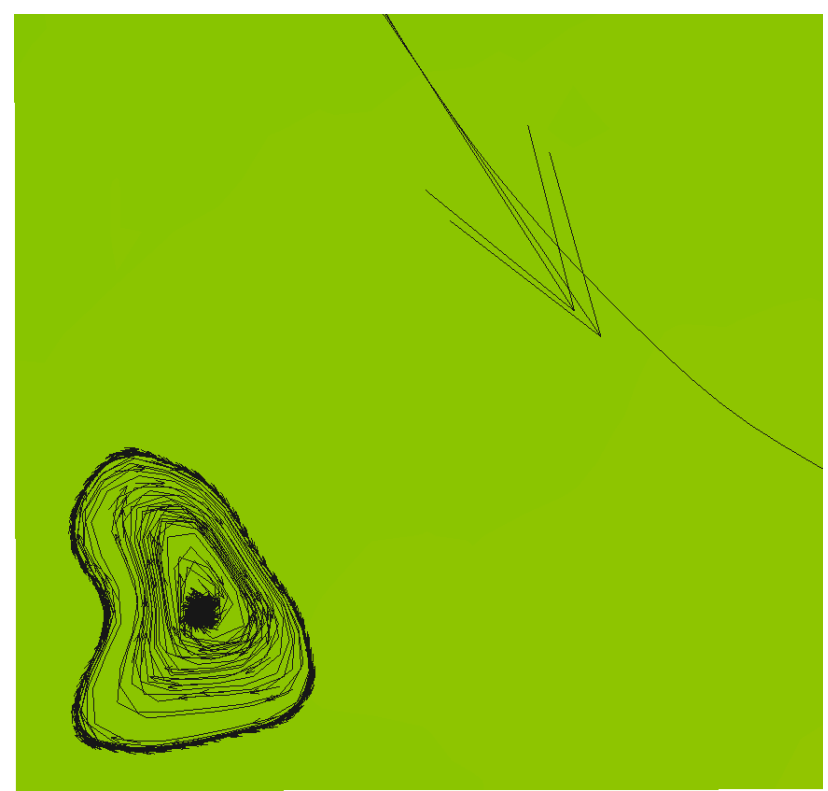

Figure 7: Enlarged view of the separated flow in the corner region. 


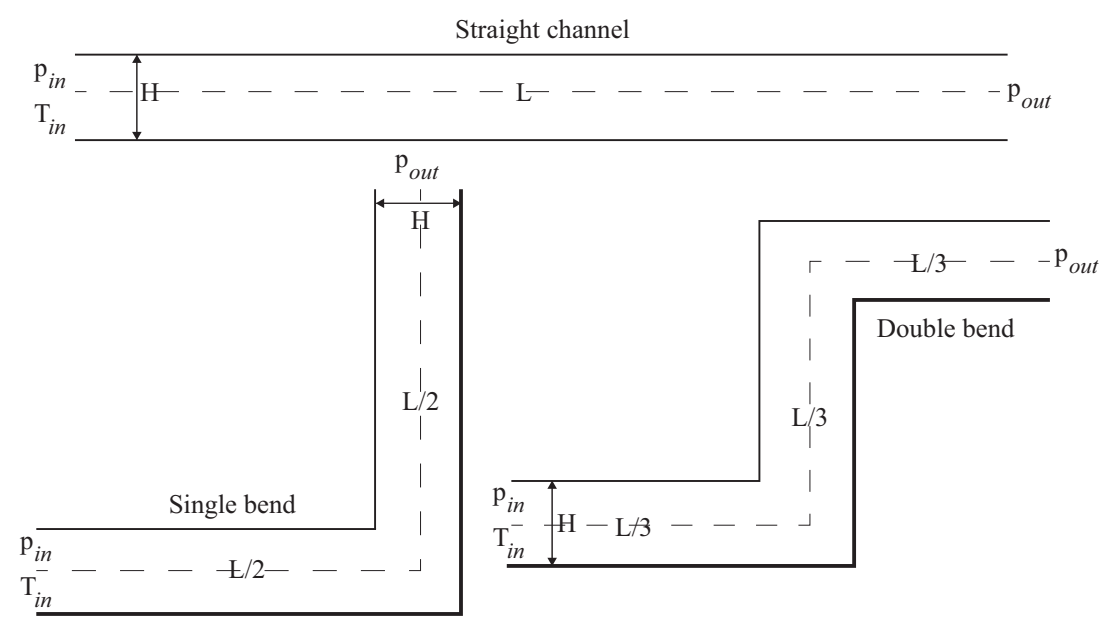

Figure 8: Schematics of the three different geometries considered. The thicker lines on the single and double bend channels represent the "lower walls" and the thinner lines are the "upper" walls; $L=15 \mu \mathrm{m}, H=1 \mu \mathrm{m}$. 


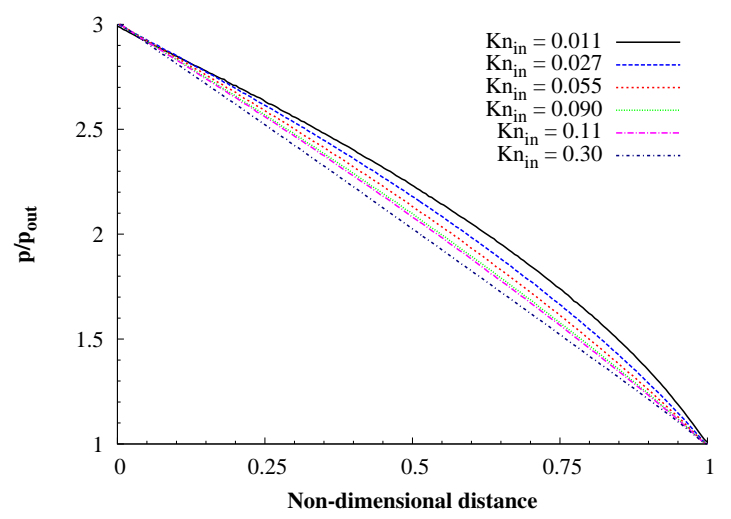

(a)

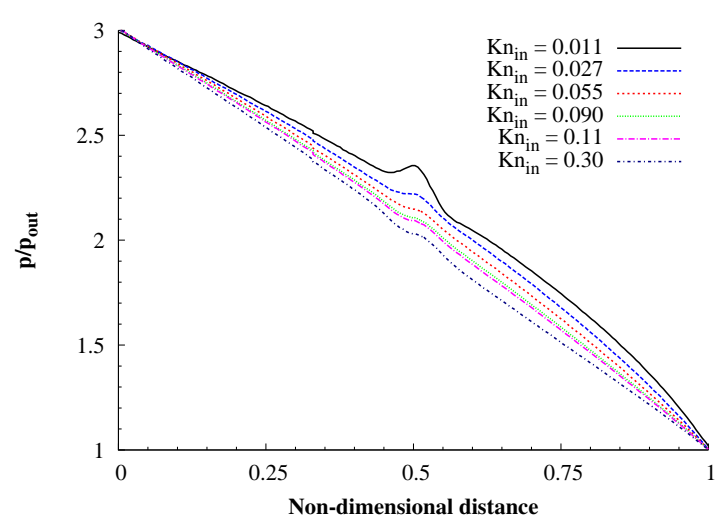

(b)

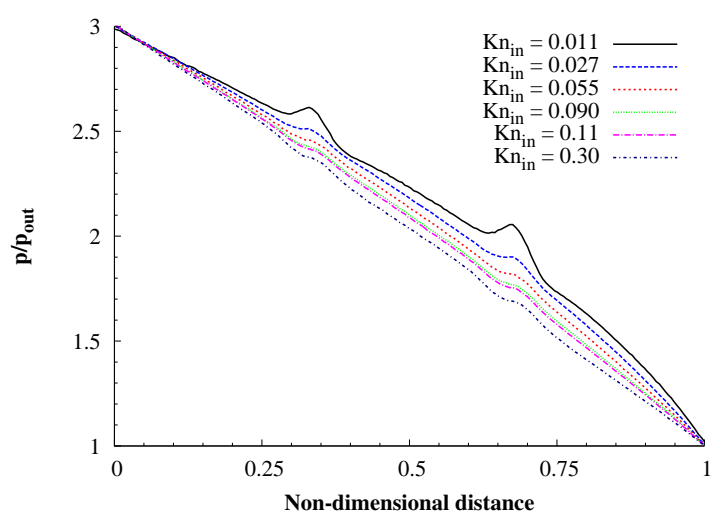

(c)

Figure 9: Streamwise centre-line pressure profiles: (a) straight channel, (b) channel with single bend, and (c) channel with two bends. 


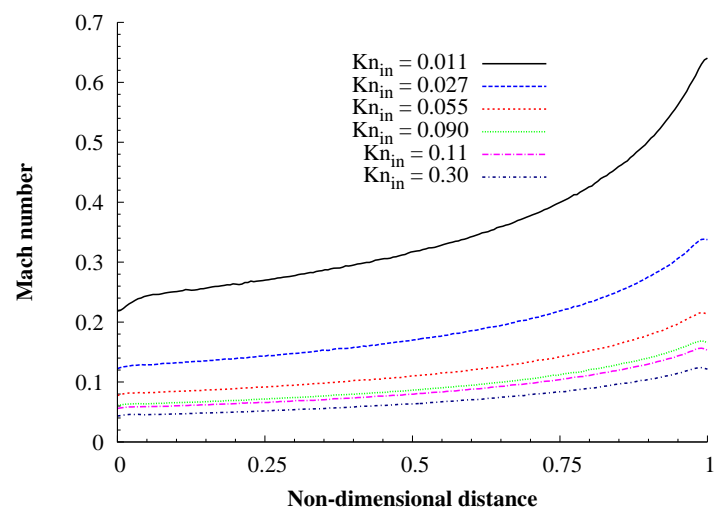

(a)

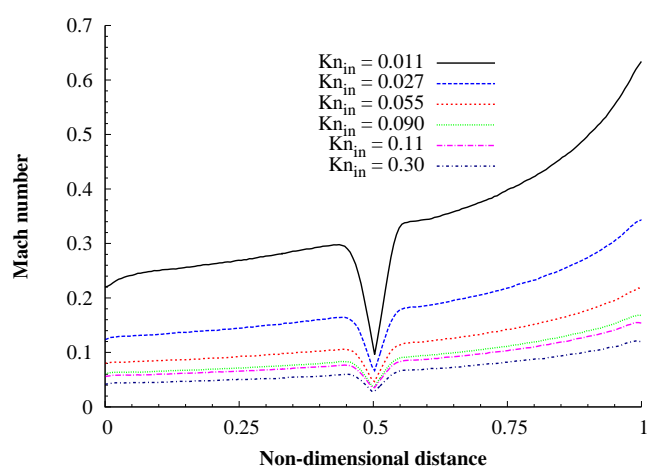

(b)

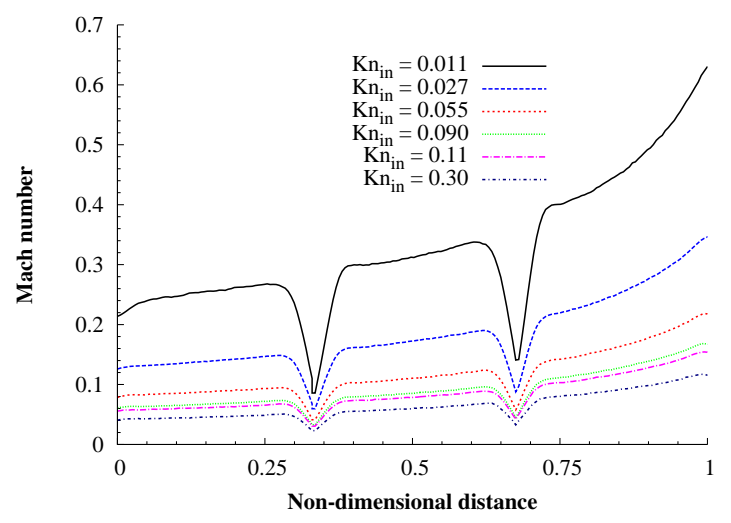

(c)

Figure 10: Streamwise centre-line Mach number profiles: (a) straight channel, (b) channel with single bend, and (c) channel with two bends. 


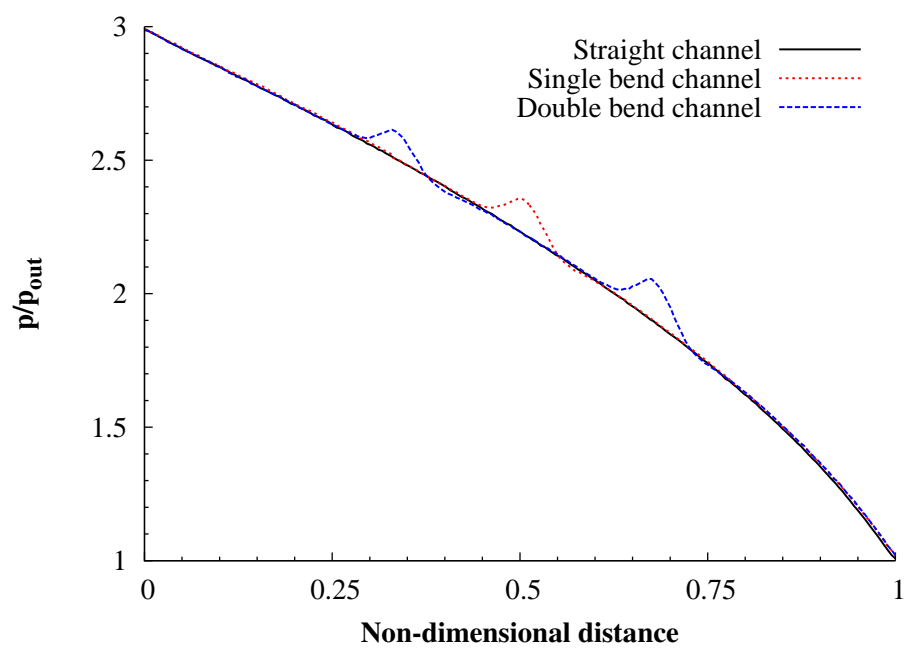

(a)

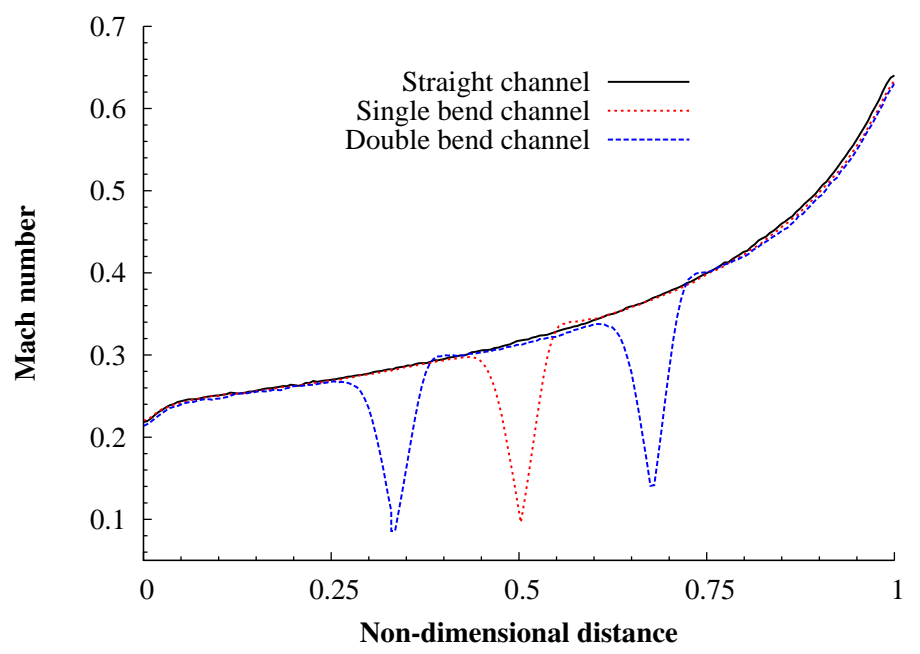

(b)

Figure 11: Comparison of centre-line profiles for the three different channel geometries at $K n_{i n}=0.011$ : (a) pressure, and (b) Mach number. 


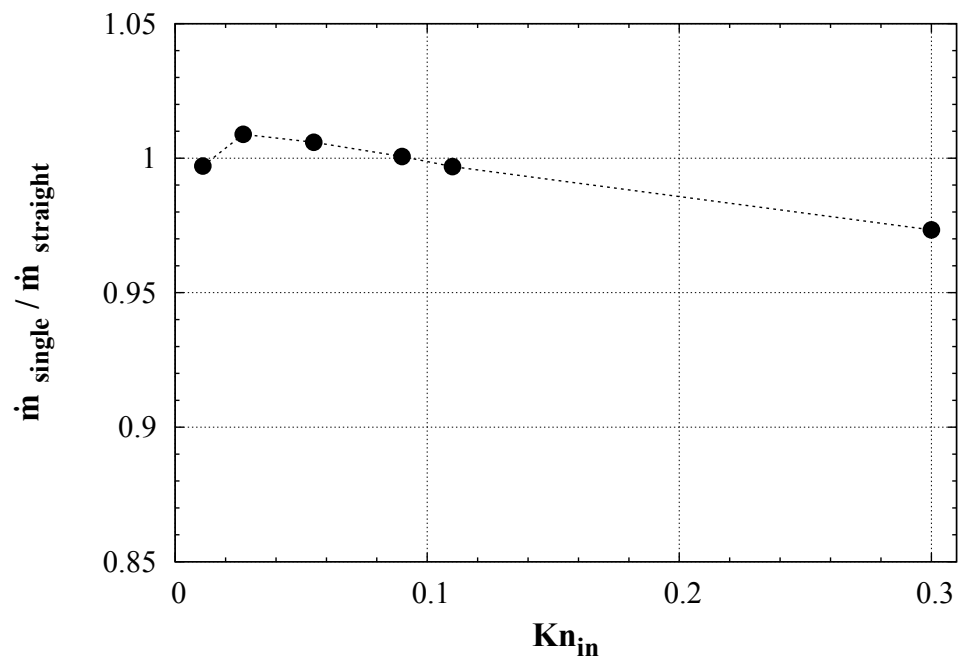

(a)

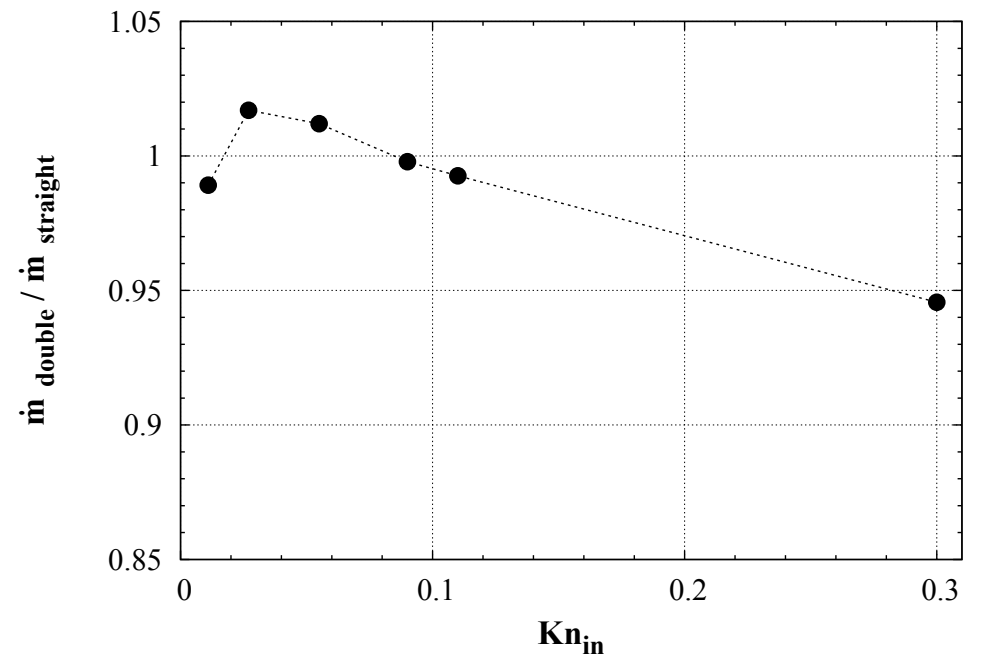

(b)

Figure 12: Mass flux measurements in micro-channels; (a) single bend, and (b) two bends. Values of mass flux are normalised by the mass flux taken in the corresponding straight channel 


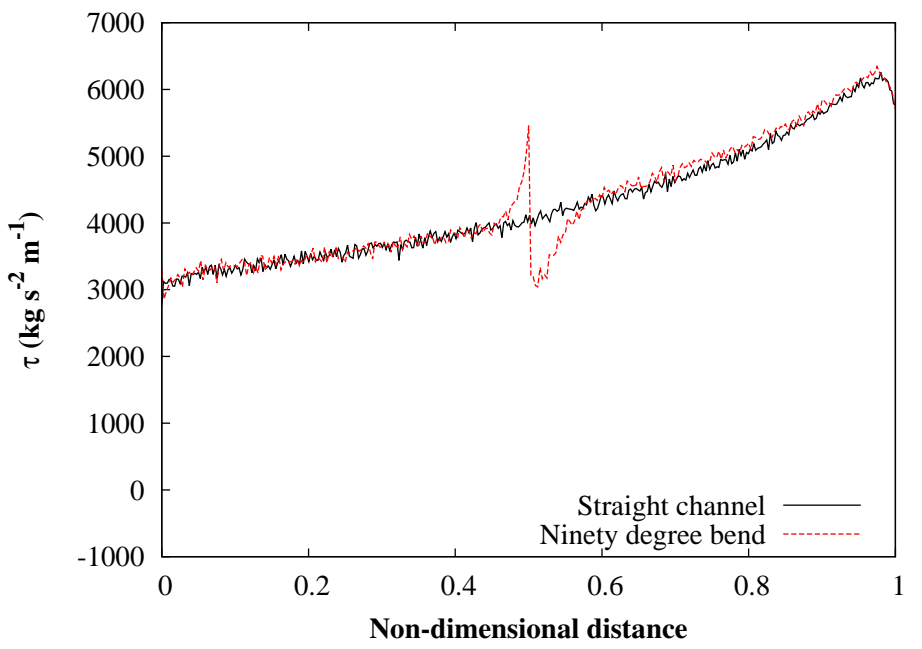

(a)

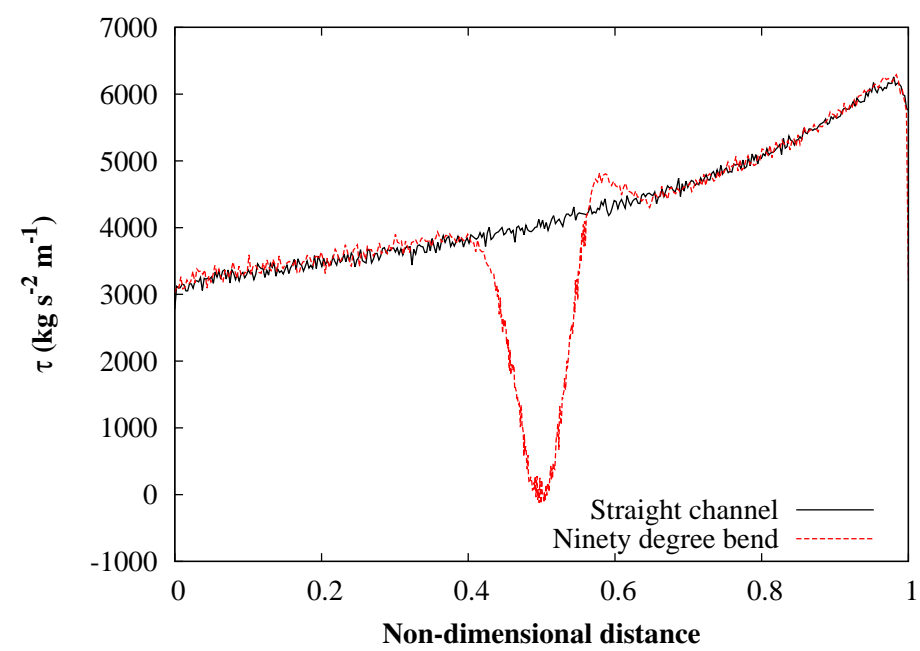

(b)

Figure 13: Shear stress at the walls of the straight and the single bend channel for $K n_{\text {in }}=0.027$; (a) upper wall, and (b) lower wall. 


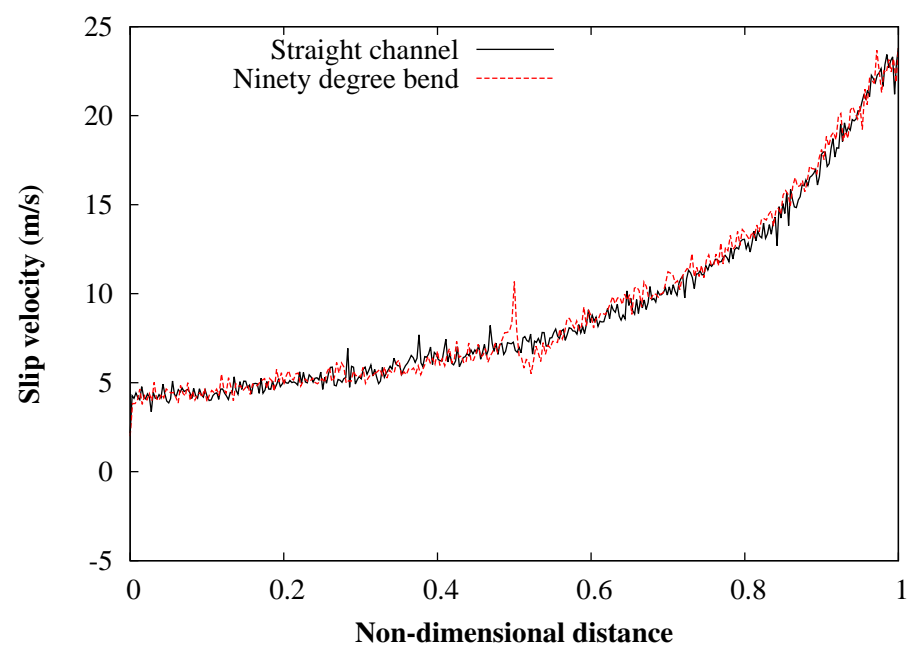

(a)

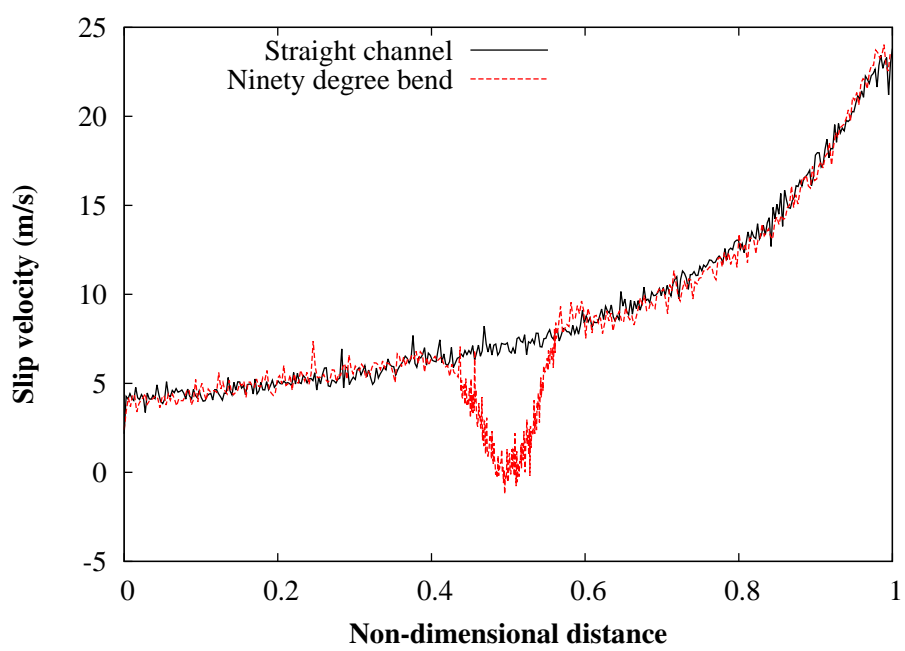

(b)

Figure 14: Slip velocity at the walls of the straight and the single bend channel for $K n_{i n}=0.027$; (a) upper wall, and (b) lower wall. 


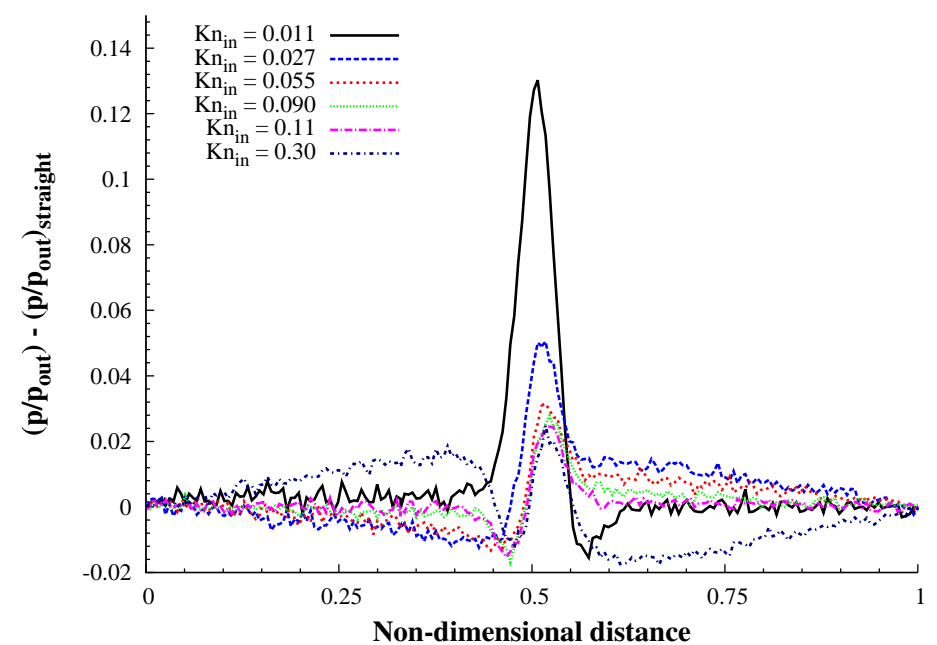

(a)

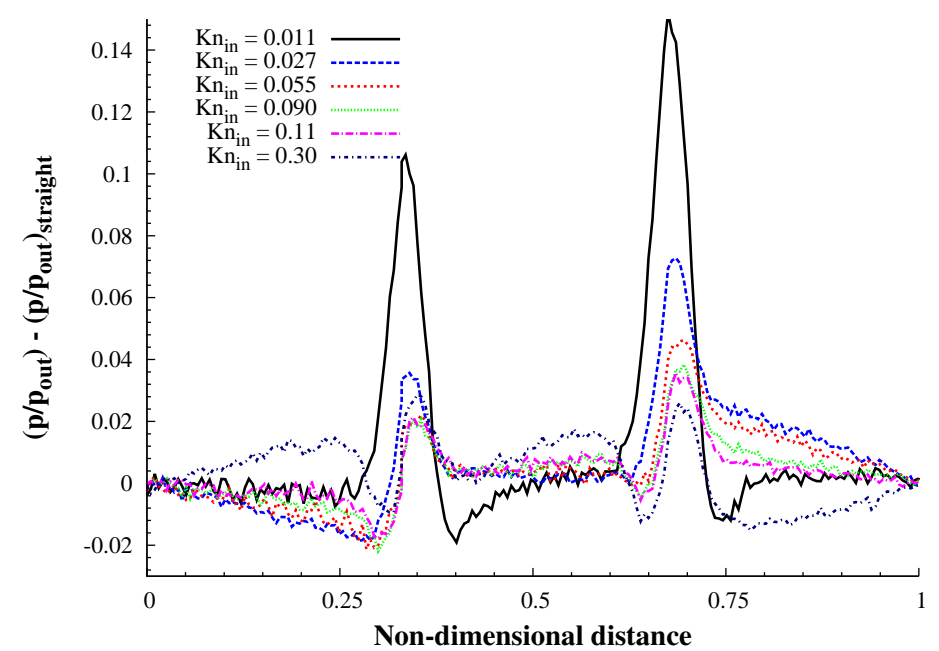

(b)

Figure 15: Differences from normalised straight-channel streamwise pressure profiles along the centre-line for (a) single bend channel, and (b) double bend channel. 
Table 1: Micro-manifold flow simulation details.

\begin{tabular}{ccccc}
\hline Case & $p_{i}(\mathrm{kPa})$ & $p_{e 1}(\mathrm{kPa})$ & $p_{e 2}(\mathrm{kPa})$ & $p_{i}: p_{e 1}: p_{e 2}$ \\
\hline I & 26.79 & 8.93 & 8.93 & $3: 1: 1$ \\
II & 26.79 & 8.93 & 17.86 & $3: 1: 2$ \\
III & 26.79 & 17.86 & 8.93 & $3: 2: 1$ \\
\hline
\end{tabular}


Table 2: Mass flux results for the micro-manifold flow cases; dsmcFoam results in bold text, Wu \& Tseng results in plain text.

\begin{tabular}{cccccccc}
\hline Case & $p_{i}: p_{e 1}: p_{e 2}$ & $\left(\dot{m}_{i} / \dot{m}_{i, I}\right)$ & $\left(\dot{m}_{i} / \dot{m}_{i}\right)$ & $\left(\dot{m}_{e 1} / \dot{m}_{i}\right)$ & $\left(\dot{m}_{e 2} / \dot{m}_{i}\right)$ & Summation & Error (\%) \\
\hline I & $3: 1: 1$ & 1.00 & 1.00 & 0.541 & 0.465 & 1.006 & 0.60 \\
I & $\mathbf{3}: \mathbf{1 : 1}$ & $\mathbf{1 . 0 0}$ & $\mathbf{1 . 0 0}$ & $\mathbf{0 . 5 3 4}$ & $\mathbf{0 . 4 6 6}$ & $\mathbf{0 . 9 9 9 9}$ & $\mathbf{- 0 . 0 0 2}$ \\
\hline II & $3: 1: 2$ & 0.755 & 1.00 & 0.981 & 0.028 & 1.009 & 0.90 \\
II & $\mathbf{3}: \mathbf{1}: \mathbf{2}$ & $\mathbf{0 . 7 5 8}$ & $\mathbf{1 . 0 0}$ & $\mathbf{0 . 9 5 8}$ & $\mathbf{0 . 0 4 2}$ & $\mathbf{0 . 9 9 9 9}$ & $\mathbf{- 0 . 0 0 5}$ \\
\hline III & $3: 2: 1$ & 0.751 & 1.00 & 0.098 & 0.910 & 1.008 & 0.80 \\
III & $\mathbf{3 : 2 : 1}$ & $\mathbf{0 . 7 4 4}$ & $\mathbf{1 . 0 0}$ & $\mathbf{0 . 0 8 7 8}$ & $\mathbf{0 . 9 1 2}$ & $\mathbf{0 . 9 9 9 9}$ & $\mathbf{- 0 . 0 0 9 5}$ \\
\hline
\end{tabular}


Table 3: DSMC simulation case details for micro-channels with and without bends.

\begin{tabular}{cccccccc}
\hline & $p_{\text {in }}(\mathrm{Pa})$ & $p_{\text {out }}(\mathrm{Pa})$ & Time step $(\mathrm{s})$ & $K n_{\text {in }}$ & $K n_{\text {out }}$ & $R e_{\text {out }}$ & $M a_{\text {out }}$ \\
\hline Case 1 & 500,000 & 166,666 & $3 \times 10^{-12}$ & 0.011 & 0.029 & 27.943 & 0.640 \\
Case 2 & 200,000 & 66,666 & $6 \times 10^{-12}$ & 0.027 & 0.078 & 5.411 & 0.337 \\
Case 3 & 100,000 & 33,333 & $6 \times 10^{-12}$ & 0.055 & 0.158 & 1.686 & 0.213 \\
Case 4 & 61,000 & 20,333 & $6 \times 10^{-12}$ & 0.090 & 0.260 & 0.792 & 0.166 \\
Case 5 & 50,000 & 16,666 & $6 \times 10^{-12}$ & 0.110 & 0.317 & 0.604 & 0.153 \\
Case 6 & 18,500 & 6,166 & $6 \times 10^{-12}$ & 0.300 & 0.862 & 0.176 & 0.122 \\
\hline
\end{tabular}


Table 4: Normalised average shear stresses in the micro-channels with bends, relative to that in the straight channel.

\begin{tabular}{ccc}
\hline \multicolumn{2}{c}{$\tau / \tau_{\text {straight }}$} \\
\hline$K n_{\text {in }}$ & Single bend & Double bend \\
\hline 0.011 & 0.8762 & 0.7567 \\
0.027 & 0.8818 & 0.8027 \\
0.055 & 0.8843 & 0.8065 \\
0.09 & 0.8980 & 0.7991 \\
0.11 & 0.8761 & 0.7962 \\
0.30 & 0.8645 & 0.7702 \\
\hline
\end{tabular}

